\title{
AN ISOPERIMETRIC PROBLEM WITH LATTICE POINT CONSTRAINTS
}

\author{
J. R. ARKINSTALL and P. R. SCOTT \\ (Received 27 August 1977; revised 23 May 1978) \\ Communicated by J. Pitman
}

\begin{abstract}
The isoperimetric problem in the Euclidean plane is completely solved for bounded, convex sets which are symmetric about the origin, and which contain no non-zero point of the integral lattice.
\end{abstract}

Subject classification (Amer. Math. Soc. (MOS) 1970): 52 A 40, 52 A 10, 10 E 05.

Let $S$ be a bounded convex set in $E^{2}$ having area $A(S)$ and perimeter $P(S)$. Let $\mathscr{S}$ denote the set of all such sets which are symmetric in the origin $O$, and which contain no non-zero point of the integral lattice. Write

$$
A_{P}(\mathscr{S})=\max _{\substack{S \in S \\ P(S)=P}} A(S) .
$$

The existence of $A_{P}(\mathscr{S})$ is assured by a simple application of the Blaschke selection theorem (see Eggleston (1963), p. 64).

THEOREM.

(a) If $0 \leqslant P \leqslant 2 \pi$, then $A_{P}(\mathscr{S})=P^{2} / 4 \pi$.

(b) If $2 \pi \leqslant P \leqslant 4+\pi$, then $A_{P}(\mathscr{S})=4-(8-P)^{2} / 4(4-\pi)$.

(c) If $4+\pi \leqslant P \leqslant 2 \sqrt{(2)}(4+\pi) /(1+\sqrt{3})$, then $A_{P}(\mathscr{S})=4-\left[8(4+\pi)-P^{2}\right] / 4(4+\pi)$.

(d) If $2 \sqrt{ }(2)(4+\pi) /(1+\sqrt{ } 3) \leqslant P \leqslant 2 \sqrt{ }(2)(1+\sqrt{ } 3)$, then

$$
A_{P}(\mathscr{S})=4-[2 \sqrt{(2)}(1+\sqrt{ } 3)-P]^{2} / 4(2 \sqrt{ }(3)-\pi) .
$$

(e) If $2 \sqrt{ }(2)(1+\sqrt{ } 3) \leqslant P$, then $A_{P}(\mathscr{S})=4$. 
The result in (a) is a restatement of the classical isoperimetric problem, since for $0 \leqslant P \leqslant 2 \pi$, the circular disc having centre $O$ and perimeter $P$ is a member of $\mathscr{S}$. Minkowski's classical theorem (Minkowski (1896)) gives the upper bound 4 for $A_{P}(\mathscr{S})$. The result in (e) gives those values of $P$ for which this bound is attained.

The proof of the theorem can be greatly simplified using Steiner symmetrization. Let $S$ be a given set, and $l$ a given line in the plane. We construct a new set $S^{\prime}$ which is symmetric about $l$ as follows. For each line $p$ orthogonal to $l$, replace the segment $p \cap S$ by a congruent segment of $p$ having its mid point on $l$. Then $S^{\prime}$ is the union of those translated segments. It is well known that Steiner symmetrization preserves convexity, area and central symmetry, does not increase perimeter, and actually decreases perimeter unless $\mathscr{S}$ is already symmetric in a line parallel to $l$. (See, for example, Eggleston (1963), p. 90.) For $S \in \mathscr{S}$, we use the notation $d(S), \partial S$ for the diameter and boundary of $S$ respectively. Let $U$ be the convex hull of the points $\pm(1, \pm 1)$, and let $Q$ be the convex hull of $\pm(2,0), \pm(0,2)$ (Fig. 1$)$.

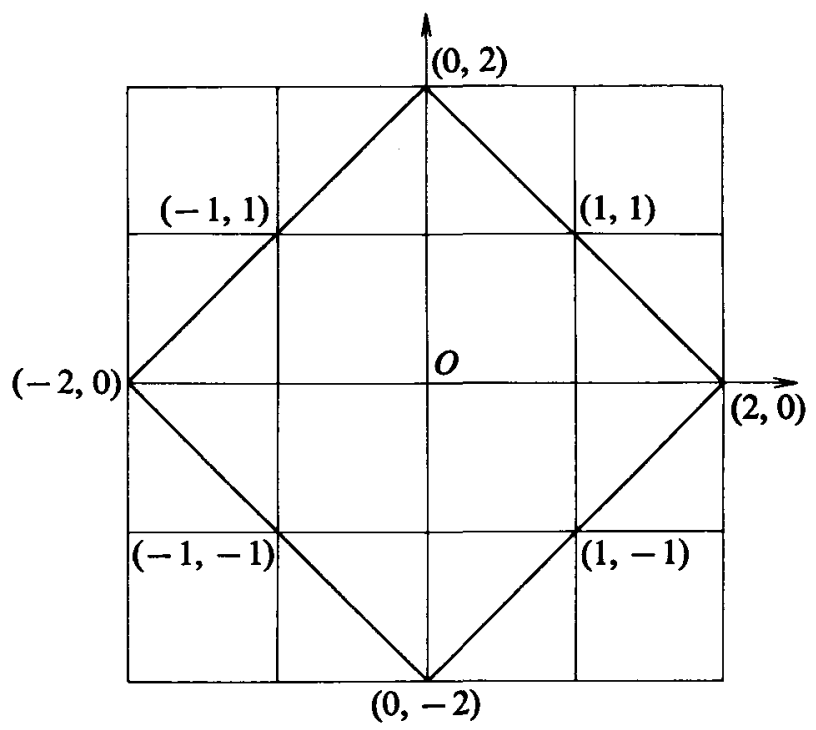

Fig. 1

LEMma. Let $S \in \mathscr{S}$ be a set for which $d(S)<4$. Then there exists a set $S^{\prime} \in \mathscr{S}$ which satisfies the following

(a) $S^{\prime} \subseteq Q$.

(b) $S^{\prime}$ is symmetric in the lines $y= \pm x$. 
(c) $A\left(S^{\prime}\right)=A(S), P\left(S^{\prime}\right) \leqslant P(S)$ and $P\left(S^{\prime}\right)=P(S)$ only if $S^{\prime}=S$.

(d) $S^{\prime} \subset H$, where $H \in \mathscr{S}$ is a hexagon, and $H=Q \cap L$ where $L$ is a parallelogram, symmetric in the lines $y= \pm x$, with sides passing through the points $\pm(1,0)$, $\pm(0,1)$.

Proor. We first show that $S$ extends beyond $Q$ in at most two quadrants of the plane. Let $q$ be a point of $S \sim Q$ in the first quadrant. By reflecting $S$ in the line $y=x$ if necessary, we may assume that $q$ lies in the second octant of the plane. Now since $q$ lies in the half-plane $y \geqslant 1$, and $(0,1)$ is not interior to $S$, it follows by convexity that any point $r$ of $S \sim Q$ in the second quadrant must lie in the halfplane $y \leqslant 1$, and so in the fourth octant of the plane. Denoting the point $(-1,1)$ by $r^{\prime}$, we see that

$$
A(\Delta O q r)>A\left(\Delta O q r^{\prime}\right)>1 \text {. }
$$

Hence the parallelogram contained in $S$ and having vertices $\pm q, \pm r$ has area greater than 4. This contradicts the bound on $A(S)$ given by Minkowski's theorem.

By reflecting $S$ in the $y$-axis if necessary, we may assume then that $S$ does not extend beyond $Q$ in the second and fourth quadrants of the plane. In fact, since $d(S)<4$ and $S$ is symmetric in $O, S$ lies in the strip $|x-y| \leqslant 2$.

We now obtain a new set $S^{\prime}$ by symmetrizing $S$ in the line $y=-x$. The lines $y=x+1, y=x, y=x-1$ intercept the interior of $S$ (and so the interior of $S^{\prime}$ ) in segments of length at most $\sqrt{2}, 2 \sqrt{2}, \sqrt{2}$ respectively. Since $S$ lies in the strip $|x-y| \leqslant 2$ it follows that $S^{\prime}$ contains no non-zero lattice points in its interior.

By the properties of Steiner symmetrization, $S^{\prime} \in \mathscr{S}, A\left(S^{\prime}\right)=A(S)$, and $P\left(S^{\prime}\right) \leqslant P(S)$ with equality only if $S^{\prime}=S$. Since $S^{\prime}$ is both centrally symmetric in $O$ and symmetric in $y=-x$, it is symmetric in $y=x$. This symmetry of $S$ together with its convexity and the fact that $\pm(1,1)$ are not interior to $S^{\prime}$, enables us to deduce that $S^{\prime} \subset Q$. Further, $S^{\prime}$ does not contain $\pm(1,0)$ or $\pm(0,1)$ as interior points; hence $S^{\prime}$ is contained in a parallelogram whose sides pass through $\pm(1,0)$, $\pm(0,1)$, and whose diagonals lie along the lines $y= \pm x$. Finally, $S^{\prime} \subseteq Q \cap L=H$, and $H \in \mathscr{S}$. This completes the proof of the lemma.

By reflecting $S^{\prime}$ in the $y$-axis, if necessary, we may assume that $\partial H$ has nonnegative slope at $(0,1)$. Let the angles common to the hexagon $H$ and the parallelogram $L$ measure $2 \psi$. We can then denote these figures explicitly by $H(\psi), L(\psi)$ respectively, where $\frac{1}{4} \pi \leqslant \psi \leqslant \frac{1}{2} \pi$ (Fig. 2). 


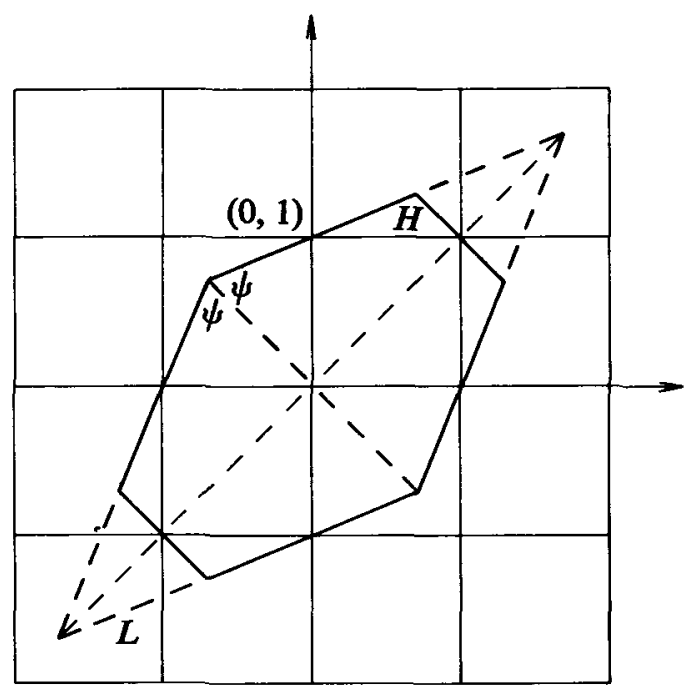

Fig. 2

We now establish part (e) of the theorem.

Let $S \in \mathscr{S}$ be a set with $A(S)=4$. We show that $P(S) \geqslant 2 \sqrt{ }(2)(1+\sqrt{ } 3)(\approx 7.73)$. Let us assume that $P(S)<8$. Then $d(S)<4$, and by the above lemma, there exists a set $S^{\prime} \in \mathscr{S}$ where $A\left(S^{\prime}\right)=4, P\left(S^{\prime}\right) \leqslant P(S)$ and $S^{\prime}$ is contained in a hexagon $H(\psi)$. In fact, since $H(\psi)$ has area $4, S^{\prime}=H(\psi)$. The perimeter of $H(\psi)$ is easily verified to be $4 \sqrt{ } 2 \operatorname{cosec} \psi+2 \sqrt{ }(2)(1-\cot (\psi))$. By elementary calculus, this function assumes its minimal value of $2 \sqrt{ }(2)(1+\sqrt{3})$ when $\psi=\frac{1}{3} \pi$; that is, when $H(\psi)$ is an equiangular hexagon. Hence $P(S) \geqslant P\left(S^{\prime}\right) \geqslant 2 \sqrt{ }(2)(1+\sqrt{ } 3)$ as required.

It remains to be shown that for each value of $P$ in this range, there is a set $S \in \mathscr{S}$ having perimeter $P$ and area 4 . For $P \geqslant 8$ we obtain $S$ by suitably shearing the square $U$ parallel to the $x$-axis. Also, over the interval $\frac{1}{4} \pi \leqslant \psi \leqslant \frac{1}{3} \pi$, the perimeter of $H(\psi)$ is a continuous function of $\psi$. Since $H\left(\frac{1}{4} \pi\right)=U$, we deduce that for each value of $P, 2 \sqrt{ }(2)(1+\sqrt{ } 3) \leqslant P \leqslant 8$, there is a hexagon having perimeter $P$ and area 4 . We conclude that for $2 \sqrt{ }(2)(1+\sqrt{3}) \leqslant P, A_{P}(\mathscr{S})=4$.

Let $S \in \mathscr{S}$ be a set for which $A(S)=A_{P}(\mathscr{S})$ where $P(S)=P<2 \sqrt{ }(2)(1+\sqrt{ } 3)$. We show that $S$ must satisfy the conditions of the set $S^{\prime}$ in the lemma (Section 2).

Since $d(S)<4$, by the above lemma there exists a set $S^{\prime} \in \mathscr{S}$ for which $A\left(S^{\prime}\right)=A(S), P\left(S^{\prime}\right) \leqslant P(S)$, and such that $S^{\prime}$ is contained in a hexagon $H(\psi)=H$. 
Now define $S(\lambda)=\lambda H+(1-\lambda) S^{\prime}(0 \leqslant \lambda \leqslant 1)$. Then $S^{\prime} \subset S(\lambda) \subset H$, and since the perimeter of $S(\lambda)$ is a continuous function of $\lambda$, there exists a set $S^{*}=S\left(\lambda^{*}\right)$ $\left(0 \leqslant \lambda^{*} \leqslant 1\right)$ for which $P\left(S^{*}\right)=P(S)$. If $\lambda>0, A\left(S^{*}\right)>A\left(S^{\prime}\right)=A(S)$, contradicting our choice of $S$. Hence $\lambda=0$, and $S=S^{\prime}$, as required.

Since $H(\psi)$ contains no non-zero lattice points in its interior, $S$ is the set of largest area and perimeter $P$ contained in $H(\psi)$. We use a result of Besicovitch (1952) which characterizes $S$, and shows that $S \in \mathscr{S}$.

Besicovitch (1952), pp. 45-48, solves the problem of finding a convex set $\Gamma$ with given perimeter and largest possible area, where $\Gamma$ is contained in a given bounded closed convex set. In our case, where the given convex set is $H(\psi)$, the required set $\Gamma$ is 'the sum point set' of all circles of some fixed radius $r$, contained in $H(\psi)$. In simple terms, the set $\Gamma$ is the hexagon $H(\psi)$ with its corners rounded off by circular arcs of common radius. Since the two sides of $H(\psi), \partial Q \cap \partial H(\psi)$, are not longer than the four equal sides of $H(\psi)$, this rounding can occur in one of only two ways. Either each corner is rounded individually (as when the circular arcs have small radius), or each of the short sides is rounded off completely by a single circular arc. In the latter case, $L(\psi)$ has effectively been rounded at each corner, using arcs of some common radius.

We formulate these cases together. A polygon $G$ is rounded at each vertex by arcs of fixed radius $r$, to form the rounded polygon $G_{r}$. We assume that $r$ is small enough for each side of $G$ to contribute a line segment to $G_{r}$. We number the vertices of $G$, and let $2 \psi_{i}$ be the internal angle at the $i$ th vertex. It is now easily seen that

$$
\begin{aligned}
& A\left(G_{r}\right)=A(G)-r^{2}\left(\sum_{i} \cot \psi_{i}-\pi\right)=A(G)-r^{2} k(G) \text { say, } \\
& P\left(G_{r}\right)=P(G)-2 r k(G) .
\end{aligned}
$$

Solving equation (2) for $r$, and substituting in equation (1) gives

$$
A\left(G_{r}\right)=A(G)-\left[P(G)-P\left(G_{r}\right)\right]^{2}[4 k(G)]^{-1} .
$$

When $G_{r}$ is obtained from $G$ by rounding, equation (3) enables us to find the maximum value of $A\left(G_{r}\right)$ when $P\left(G_{r}\right)$ is a given constant. Our problem is complicated by the fact that $G=H(\psi)$ or $G=L(\psi)$ are themselves dependent on a 
parameter $\psi$. To find the maximum value of $A\left(G_{r}\right)$ we need to examine the derivative $A\left(G_{r}\right)$ with respect to $\psi$ :

$$
\frac{d A\left(G_{r}\right)}{d \psi}=\frac{d A(G)}{d \psi}-\frac{2\left[P(G)-P\left(G_{r}\right)\right]}{4 k(G)} \frac{d P(G)}{d \psi}+\frac{\left[P(G)-P\left(G_{r}\right)\right]^{2}}{4[k(G)]^{2}} \frac{d k(G)}{d \psi} .
$$

Let $\psi$ be given. According to Besicovitch's construction, there will be a certain value $P_{0}(\psi)$ of the perimeter for which $H_{r}(\psi)$ and $L_{r}(\psi)$ coincide. For $P<P_{0}(\psi)$, it will not be possible to construct $H_{r}(\psi)$, there being insufficient perimeter to reach the sides of $H(\psi)$ through $\pm(1,1)$. For $P>P_{0}(\psi), L_{r}(\psi)$ will contain $\pm(1,1)$ as interior points, and will thus no longer be a member of $\mathscr{S}$.

We show that

$$
P_{0}(\psi)=P(G)-k(G) \frac{\sqrt{2}(1-\tan \psi)}{1-\sec \psi},
$$

where $G=L(\psi)$.

Let $G_{r}$ be the rounded parallelogram with perimeter $P_{0}(\psi)$. Then the points $\pm(1,1)$ lie on the boundary $\partial G_{r}$, the diameter of $G_{r}$ lies along the line $y=x$, and $d\left(G_{r}\right)=2 \sqrt{2}$. We calculate $d\left(G_{r}\right)$ using the construction of Fig. 3 .

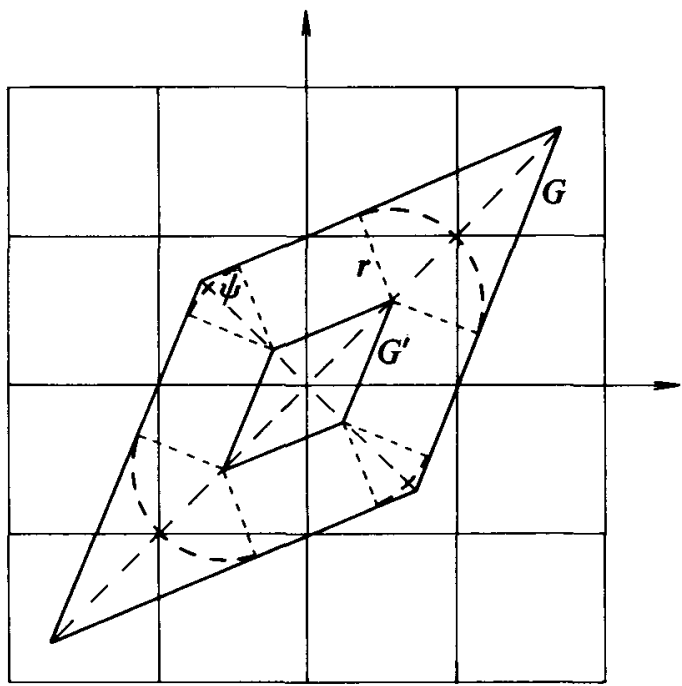

Fig. 3 
We note that the parallelogram $G$ has diameter $2 \sin \left(\frac{3}{4} \pi-\psi\right) \cdot \sec \psi$, and that the similar parallelogram $G^{\prime}$ is obtained from $G$ by an enlargement of scale factor $1-r \sec \left(\psi-\frac{1}{1} \pi\right)$. Now

$$
\begin{aligned}
d\left(G_{r}\right) & =2 r+2 \sin \left(\frac{\pi}{2} \pi-\psi\right) \cdot \sec \psi\left[1-r \sec \left(\psi-\frac{1}{4} \pi\right)\right] \\
& =2 r(1-\sec \psi)+\sqrt{ }(2)(1+\tan \psi) .
\end{aligned}
$$

Equating this to $2 \sqrt{ }$, solving for $r$, and substituting in (2) gives the above value for $P_{0}(\psi)$, for $\frac{1}{4} \pi \leqslant \psi<\frac{1}{2} \pi$. $P_{0}\left(\frac{1}{2} \pi\right)$, by definition, equals $\sqrt{ }(2)(2+\pi)$, making $P_{0}(\psi)$ continuous on $\left[\frac{1}{4} \pi, \frac{1}{2} \pi\right]$. It may be demonstrated that $P_{0}\left(\frac{1}{2} \pi\right)$ is the least value which $P_{0}(\psi)$ takes. A particular value of interest is $P_{0}\left(\frac{1}{3} \pi\right)=2 \sqrt{ }(2)(4+\pi) /(1+\sqrt{3})$.

We now apply the results of Section 5 to our problem.

Case 1. Suppose $G=H(\psi)$. By straightforward calculation we find that

$$
\begin{aligned}
A(G) & =4, \\
P(G) & =2 \sqrt{ }(2)(2 \operatorname{cosec} \psi+1-\cot \psi), \\
k(G) & =2 \cot \psi+4 \tan \frac{1}{2} \psi-\pi \\
& =\frac{1}{2} \sqrt{ }(2) P(G)-(2+\pi) \\
& >0, \text { since } P(G) \geqslant 2 \sqrt{ }(2)(1+\sqrt{ } 3) .
\end{aligned}
$$

Substituting in equation (4) and setting $P\left(G_{r}\right)=P$,

$$
\begin{aligned}
\frac{d A\left(G_{r}\right)}{d \psi} & =\left[\frac{P(G)-P}{2 \sqrt{ }(2) k(G)}\right]\left[-\sqrt{ } 2 \frac{d P(G)}{d \psi}+\left(\frac{P(G)-P}{\sqrt{ }(2) k(G)}\right) \frac{d k(G)}{d \psi}\right] \\
& =\left[\frac{P(G)-P}{2 \sqrt{(2) k(G)}}\right] \cdot \frac{d P(G)}{d \psi} \cdot\left[-\sqrt{ } 2+\left(\frac{P(G)-P}{2 k(G)}\right)\right] \\
& =2 r(1-2 \cos \psi) \operatorname{cosec}^{2} \psi(r-\sqrt{ } 2) .
\end{aligned}
$$

Clearly $r-\sqrt{2}<0$, since circles of radius $\sqrt{2}$ cannot be contained in $G$. Since we are assuming $2 \pi \leqslant P \leqslant 2 \sqrt{ }(2)(1+\sqrt{ } 3)$, the first term is non-negative for all $\psi$, and zero only when $\psi=\frac{1}{3} \pi$. Finally, $1-2 \cos \psi\left(\frac{1}{4} \pi \leqslant \psi \leqslant \frac{1}{2} \pi\right)$ is an increasing function of $\psi$ with a zero at $\psi=\frac{1}{3} \pi$. Hence for any value of $P, A\left(G_{r}\right)$ assumes its maximal value when and only when $\psi=\frac{1}{\mathrm{~g}} \pi$. 
Case 2. Suppose now that $G=L(\psi)$. Setting $a=\tan \psi+\cot \psi$ we find that

$$
\begin{aligned}
A(G) & =a+2, \\
P(G) & =2 \sqrt{ }(2)(\operatorname{cosec} \psi+\sec \psi) \\
& =2 \sqrt{ }(2) a^{ \pm}(a+2)^{\ddagger}, \\
k(G) & =2 a-\pi .
\end{aligned}
$$

Hence from equation (4), setting $P\left(G_{r}\right)=P$,

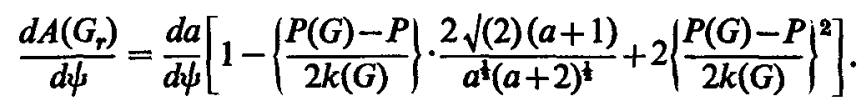

Solving this quadratic expression in $[P(G)-P] / 2 k(G)$, and simplifying gives

$$
\frac{d A\left(G_{r}\right)}{d \psi}=\frac{d a}{d \psi} \frac{1}{2[k(G)]^{2}}\left[P-\sqrt{ }(2)(a+2)^{\ddagger} a^{-1} \pi\right]\left[P-\sqrt{ }(2) a^{4}(a+2)^{-1}(4+\pi)\right] .
$$

Now $d a / d \psi=\operatorname{cosec}^{2} \psi\left(\tan ^{2} \psi-1\right) \geqslant 0$ for $\frac{1}{4} \pi \leqslant \psi<\frac{1}{2} \pi$, with equality only if $\psi=\frac{1}{4} \pi$. The term $1 / 2[k(G)]^{2}$ is obviously positive. A simple calculation shows that $\sqrt{ }(2)(a+2)^{\ddagger} a^{-k} \leqslant 2$ with equality only for $\psi=1 \pi$. Since $P \geqslant 2 \pi$, the term $\left[P-\sqrt{ }(2)(a+2)^{\frac{1}{4}} a^{-\frac{1}{2}} \pi\right]$ is non-negative, and zero only when $P=2 \pi$ and $\psi=1 \pi$. Finally, for $\frac{1}{4} \pi \leqslant \psi<\frac{1}{2} \pi, \sqrt{ }(2) a^{\frac{1}{}}(a+2)^{-1}$ is an increasing function of $\psi$, assuming its minimum value of 1 when $\psi=1 \pi$. Hence, if $P \leqslant 4+\pi$,

$$
P-\sqrt{ }(2) a^{\frac{1}{}}(a+2)^{-1}(4+\pi) \leqslant P-(4+\pi) \leqslant 0 .
$$

If $P \geqslant 4+\pi$, then $P-\sqrt{ }(2) a^{\frac{1}{2}}(a+2)^{-1}(4+\pi)$ is a decreasing function of $\psi$, with a zero at $\psi=T$, corresponding to the root of

$$
P=\sqrt{ }(2) a^{t}(a+2)^{-1}(4+\pi) .
$$

Thus for $2 \pi \leqslant P<4+\pi, A\left(G_{r}\right)$ assumes its maximum value at $\psi=1 \pi$. For $4+\pi \leqslant P \leqslant 2 \sqrt{ }(2)(1+\sqrt{ } 3), A\left(G_{r}\right)$ assumes its maximum value when $\psi=T$.

We can now establish part $(b)$ of the theorem. Suppose

$$
2 \pi \leqslant P \leqslant 4+\pi<\sqrt{ }(2)(2+\pi) .
$$

By Section 6, $G$ must be a parallelogram. Hence $A\left(G_{r}\right)$ assumes its maximum when $G=L\left(\frac{1}{2} \pi\right)$, and

$$
A_{P}(\mathscr{S})=A\left(L_{\zeta}(1 \pi)\right)=4-(8-P)^{2} / 4(4-\pi) .
$$

Suppose that $4+\pi \leqslant P \leqslant 2 \sqrt{ }(2)(1+\sqrt{3})$. From Section 7 we know that the function $A\left(G_{r}(\psi)\right)$ assumes its maximum when $G=L(T)$ or when $G=H\left(\frac{1}{3} \pi\right)$. 
However, we do not yet know whether the rounded polygons $G_{r}$ can be formed for $P$ in the given range.

Let $G=L(T)$. For $P \geqslant 4+\pi$, the angle $T$ is determined by (6):

$$
P=\sqrt{ }(2)(4+\pi) a^{t}(a+2)^{-1}=\sqrt{ }(2)(4+\pi)(\sin T+\cos T)^{-1} .
$$

From (2), the radius by which $L_{T}(T)$ is rounded is given by

$$
r=(P(G)-P)[2 k(G)]^{-1} \text {. }
$$

Substituting for $P(G), k(G)$, and then for $P$ we obtain

$$
r=P /(8+2 \pi)=[\sqrt{ }(2)(\sin T+\cos T)]^{-1} .
$$

From (5), the diameter of $G_{r}$ is given by

$$
\begin{aligned}
d\left(G_{r}\right) & =2 r(1-\sec T)+\sqrt{ }(2)(1+\tan T) \\
& =\sqrt{ }(2)(1+2 \sin T)(\sin T+\cos T)^{-1}
\end{aligned}
$$

If $d\left(G_{r}\right)>2 \sqrt{2}, L_{r}(T)$ contains $\pm(1,1)$ as interior points. We find that for $\frac{1}{2} \pi \leqslant T \leqslant \frac{1}{2} \pi, d\left(G_{r}\right)>2 \sqrt{2}$ if and only if $T>\frac{1}{3} \pi$. From (7), this occurs precisely when $P>8(4+\pi) / 2 \sqrt{ }(2)(1+\sqrt{ } 3)$.

The area of $L_{r}(T)$ can be found from (3) by substituting in the value of $P$ from (6). A short calculation gives

$$
A\left(L_{r}(T)\right)=2+P^{2} / 4(4+\pi) .
$$

Again, directly from (3) we have

$$
A\left(H_{r}\left(\frac{1}{3} \pi\right)\right)=4-[2 \sqrt{ }(2)(1+\sqrt{3})-P]^{2} / 4(2 \sqrt{ }(3)-\pi) .
$$

Graphically, these two area functions of $\boldsymbol{P}$ are represented by parabolas, the first upright, and the second inverted. A lengthy but elementary calculation shows that these parabolas touch when $P=2 \sqrt{ }(2)(4+\pi) /(1+\sqrt{ } 3)$. It follows that $A\left(L_{r}(T)\right) \geqslant A\left(H_{r}\left(\frac{1}{3} \pi\right)\right)$, with equality only when $P=2 \sqrt{(2)}(4+\pi) /(1+\sqrt{ } 3)$. Since we have just shown that the construction of $L_{r}(T)$ is valid for $P \leqslant 2 \sqrt{ }(2)(4+\pi) /(1+\sqrt{ } 3)$ we have established part $(c)$ of the theorem.

Suppose now that $2 \sqrt{ }(2)(4+\pi) /(1+\sqrt{3}) \leqslant P \leqslant 2 \sqrt{ }(2)(1+\sqrt{ } 3)$. As we note in Section $6, P_{0}\left(\frac{1}{3} \pi\right)=2 \sqrt{ }(2)(4+\pi) /(1+\sqrt{ } 3)$ and so $H_{r}\left(\frac{1}{3} \pi\right)$ can be constructed for $P$ in this range. However, $L_{r}(T)$ is now disallowed, as $\pm(1,1)$ are interior points. Substituting the value of $r$ given by (2) in expression (5), we see that the diameter $d(L(\psi))$ is a continuous function of $\psi$ for $\frac{1}{2} \pi \leqslant \psi<\frac{1}{2} \pi$. It follows that for values of $\psi$ lying in a certain open interval about $T$, the rounded parallelogram $L_{r}(\psi)$ having perimeter $P$ will contain $\pm(1,1)$ as interior points. Also, the function $A\left(L_{r}(\psi)\right)$ is a 
continuous function of $\psi$ with a single (maximum) turning point at $\psi=T$. Hence the maximum allowable value of $A\left(L_{r}(\psi)\right)$ will be assumed for a rounded parallelogram $L_{r}(\psi)$ which has $\pm(1,1)$ as boundary points. But now $L_{r}(\psi)$ coincides with $H_{r}(\psi)$, and of all such rounded hexagons of perimeter $P, H_{r}\left(\frac{1}{8} \pi\right)$ has the largest area. Since $H_{r}\left(\frac{1}{3} \pi\right)$ can be constructed, we have established part $(d)$ of the theorem.

This completes the proof of the theorem.

Consider now the problem of finding

$$
h(\beta)=\max _{S \in \mathscr{S}} \frac{A(S)}{[P(S)]^{\beta}},
$$

where $0 \leqslant \beta \leqslant 2$. The existence of such a maximum is guaranteed by Blaschke's selection theorem. When $\beta=0$, the solution of this problem is given by Minkowski's theorem. When $\beta=2$, we have the classical unconstrained isoperimetric problem.

For given $\beta$, let $S^{*} \in \mathscr{S}$ be the set for which $h(\beta)$ is attained. $S^{*}$ must have the largest area of all sets of perimeter $P\left(S^{*}\right)$. Hence $S^{*}$ must be one of the sets $S$ for which $A(S)=A_{P}(\mathscr{S})$ in the above theorem. In particular, by calculating the ratios $A_{P}(\mathscr{S}) / P$ we are able to confirm the conjecture of Scott (1974):

ThEOREM. $h(1)=2(2+\sqrt{ } \pi)^{-1}$, attained when $S$ is the square with rounded corners, $L_{T}\left(\frac{1}{4} \pi\right)$, having $r=2(2+\sqrt{ } \pi)^{-1}$.

\section{References}

A. S. Besicovitch (1952), 'Variants of a classical isoperimetric problem', Quart. J. Math. Oxford (2) 3, 42-49.

H. G. Eggleston (1963), Convexity (Cambridge Tract 47).

H. Minkowski (1896), Geometrie der Zahlen (Leipzig and Berlin).

P. R. Scott (1974), ‘An area-perimeter problem', Amer. Math. Monthly 81 (8), 884-885.

Department of Pure Mathematics

University of Adelaide

South Australia 